\title{
SUMMABILITY OF A CLASS OF FOURIER SERIES
}

\section{G. M. PETERSEN}

1. In this section we shall consider a class of summability methods which sum the Fourier series

$$
\frac{1}{2} a_{0}+\sum_{k=1}^{\infty}\left(a_{k} \cos k x+b_{k} \sin k x\right)
$$

of a function $f(x)$ which is Lebesgue integrable and satisfies the condition

$$
|f(x+h)-f(x)|=o\left[\left(\log \frac{1}{|h|}\right)^{-1}\right]
$$

for some $x$. It is known that the Fourier series of such functions need not converge [3, p. 173]. The partial sums, $s_{k}(x)$, of (1) (since without loss of generality we may suppose $f(x)$ to be even) are given by:

$$
s_{k}(x)=\frac{2}{\pi} \int_{0}^{\pi} f(x+t) D_{k}(t) d t
$$

where

$$
D_{k}(t)=\frac{\sin \left(k+\frac{1}{2}\right) t}{2 \sin \frac{1}{2} t} ;
$$

$D_{k}(t)$ is called the Dirichlet kernel.

Suppose now that the matrix $A=\left(a_{n, k}\right)$ determines a regular summability method. We shall also assume that $A$ is a triangular matrix, i.e., $a_{n, k}=0$ for $k \geqq n+1$. The $A$ transforms $t_{n}(x)$ of the partial sums $\left\{s_{k}(x)\right\}$ may then be written as follows:

$$
t_{n}(x)=\sum_{k=1}^{n} a_{n, k} s_{k}(x)=\frac{2}{\pi} \sum_{k=1}^{n} a_{n, k} \int_{0}^{\pi} f(x+t) D_{k}(t) d t .
$$

Regular matrices which satisfy the condition

$$
\lim _{n \rightarrow \infty} \sum\left|a_{n, k}-a_{n, k+1}\right|=0
$$

Received by the editors August 31, 1959 and, in revised form, November 16, 1959 and December 28, 1959. 
are called strongly regular $[1 ; 2]$. We shall employ a slightly stronger condition in the theorem that follows. The proof of this theorem parallels that of a similar theorem due to Hardy and Littlewood [3, p. 34].

Theorem. If

(i) $A$ is a regular triangular matrix such that for some $0<r<1$,

$$
\lim _{n \rightarrow \infty} \sum k^{r}\left|a_{n, k}-a_{n, k+1}\right|=0,
$$

(ii) $f(t)$ is Lebesgue integrable over $(0, \pi)$ and

$$
|f(x+h)-f(x)|=o\left[\left(\log \frac{1}{|h|}\right)^{-1}\right]
$$

for some $x$ then at the point $x$, the Fourier series of $f(t)$ is summable by $A$ to $f(x)$.

Proof. Clearly we may suppose that $f(t)$ is an even function and $f(x)=0$. We then have

$$
\begin{aligned}
& \left|\sum_{k=1}^{n} a_{n, k} \int_{0}^{\pi} f(x+t) D_{k}(t) d t\right| \leqq\left|\sum_{k=1}^{n} a_{n, k} \int_{0}^{k^{-1}} f(x+t) D_{k}(t) d t\right| \\
& \quad+\left|\sum_{k=1}^{n} a_{n, k} \int_{k^{-1}}^{k^{-r}} f(x+t) D_{k}(t) d t\right|+\left|\sum_{k=1}^{n} a_{n, k} \int_{k^{-r}}^{\pi} f(x+t) D_{k}(t) d t\right| \\
& \quad=\left|\sum_{k=1}^{n} a_{n, k} P_{k}(x)\right|+\left|\sum_{k=1}^{n} a_{n, k} Q_{k}(x)\right|+\left|\sum_{k=1}^{n} a_{n, k} R_{k}(x)\right| .
\end{aligned}
$$

Since $\left|D_{k}(t)\right| \leqq M k$ in $\left(0, k^{-1}\right)$

$$
\left|P_{k}(x)\right|=\left|\int_{0}^{k^{-1}} f(x+t) D_{k}(t) d t\right| \leqq M k \int_{0}^{k^{-1}}|f(x+t)| d t \rightarrow 0
$$

as $k \rightarrow \infty$, since $f(x+t)$ is continuous at $x$. Consequently $\left\{P_{k}(x)\right\}$ is a null sequence. Also $\left|D_{k}(t)\right| \leqq M^{\prime} / t$ and

$$
\begin{aligned}
\left|Q_{k}(x)\right| & =\left|\int_{k^{-1}}^{k^{-r}} f(x+t) D_{k}(t) d t\right| \leqq M o\left[(\log |k| r)^{-1}\right] \int_{k^{-1}}^{k^{-r}}\left|\frac{d t}{t}\right| \\
& =M^{\prime} o\left[\left(\log |k|^{r}\right)^{-1}\right][\log |k|-r \log |k|] .
\end{aligned}
$$

Clearly $Q_{k}(x)$ is a null sequence and the first two sums in (3) can be made as small as we may wish by choosing $n$ sufficiently large. For some $\delta,|f(x+t)|<1$ for $0 \leqq t \leqq \delta$, and for large $k$ such that $k^{-r}>\delta$ 


$$
R_{k}(x)=\int_{k^{-r}}^{\delta} f(x+t) D_{k}(t) d t+\int_{\delta}^{\pi} f(x+t) D_{k}(t) d t=R_{k}^{\prime}(x)+R_{k}^{\prime \prime}(x) .
$$

By the Riemann-Lebesgue theorem, $\left\{R_{k}^{\prime \prime}(x)\right\}$ is a null sequence and so is $A$ summable to zero. We must now evaluate $\left|\sum a_{n, k} R_{k}^{\prime}(x)\right|$. First we introduce the notation

$$
D_{0}(t)+\cdots+D_{k}(t)=\frac{\sin ^{2}(k+1)(t / 2)}{2 \sin ^{2}(t / 2)}=K_{k}(t),
$$

and observe that $\left|K_{k}(t)\right| \leqq M^{\prime \prime} / t^{2}$. For some $k, k=1,2, \cdots, N, k^{-r}$ $\geqq \delta$. However, since $\delta$ (and hence $N$ ) is fixed,

$$
\lim _{n \rightarrow \infty} \sum_{1}^{N}\left|a_{n, k}\right|=0 .
$$

Consider the matrix $\left(b_{n, k}\right), b_{n, k}=0, k=1,2, \cdots, N, b_{n, k}=a_{n, k}$ elsewhere. The matrices $\left(b_{n, k}\right)$ and $\left(a_{n, k}\right)$ are equivalent, indeed for any sequence $\left\{s_{k}\right\}$

$$
\lim _{n \rightarrow \infty} \sum\left(a_{n, k}-b_{n, k}\right) s_{k}=0
$$

Therefore in the sequel we shall assume without any loss of generality that $a_{n, k}=0, k=1,2, \cdots, N$. We have for $n>N$

$$
\begin{aligned}
\left|\sum_{k=1}^{n} a_{n, k} R_{k}^{\prime}(x)\right|= & \left|\sum_{k=N}^{n} a_{n, k} \int_{k^{-r}}^{\delta} f(x+t)\left[K_{k}(t)-K_{k-1}(t)\right] d t\right| \\
\leqq & \left|\sum_{k=N}^{n-1}\left(a_{n, k}-a_{n, k+1}\right) \int_{k^{-r}}^{\delta} f(x+t) K_{k}(t) d t\right| \\
& +\left|\sum_{k=N+1}^{n} a_{n, k} \int_{k^{-r}}^{(k-1)^{-r}} f(x+t) K_{k}(t) d t\right| .
\end{aligned}
$$

It then follows that

$$
\begin{aligned}
\left|\sum_{k=N}^{n-1}\left(a_{n, k}-a_{n, k+1}\right) \int_{k^{-r}}^{\delta} f(x+t) K_{k}(t) d t\right| & \\
& \leqq \sum_{k=N}^{n-1}\left|a_{n, k}-a_{n, k+1}\right| \int_{k^{-r}}^{\delta}\left|\frac{M^{\prime \prime}}{t^{2}}\right| d t \\
& \leqq M^{\prime \prime} \sum_{k=N}^{n-1}\left(k^{r}+\delta^{-1}\right)\left|a_{n, k}-a_{n, k+1}\right|
\end{aligned}
$$

and it is clear from the hypothesis 


$$
\lim _{n \rightarrow \infty} \sum_{k=N}^{n-1}\left(k^{r}+\delta^{-1}\right)\left|a_{n, k}-a_{n, k+1}\right|=0 .
$$

Moreover,

$$
\begin{aligned}
&\left|\sum_{k=N+1}^{n} a_{n, k} \int_{k^{-r}}^{(k-1)^{-r}} f(x+t) K_{k}(t) d t\right| \leqq \sum_{k=N+1}^{n}\left|a_{n, k}\right|\left|\int_{k^{-r}}^{(k-1)^{-r}} \frac{M^{\prime \prime}}{t^{2}} d t\right| \\
&=M^{\prime \prime} \sum_{k=N+1}^{n}\left|a_{n, k}\right|\left[(k-1)^{r}-k^{r}\right] .
\end{aligned}
$$

Now the matrix $\left(\left|a_{n, k}\right|\right)$ is not regular but does sum null sequences to zero if $\left(a_{n, k}\right)$ is a regular matrix. The sequence $\left\{(k-1)^{r}-k^{r}\right\}$ is a null sequence for $0<r<1$ and so

$$
\lim _{n \rightarrow \infty} \sum_{k=1}^{n}\left|a_{n, k}\right|\left[k^{r}-(k+1)^{r}\right]=0
$$

and $\lim _{n \rightarrow \infty}\left|\sum_{k=1}^{n} a_{n, k} R_{k}^{\prime}(x)\right|=0$. This completes the proof of the theorem.

2. We have remarked before that there are functions of our class, even continuous functions, such that

$$
\limsup _{k \rightarrow \infty} s_{k}(x)=\infty,
$$

Consider the matrix $B=\left(b_{n, k}\right)$ where $b_{n, k}=1 / n$ for $1 \leqq k \leqq n-1$, $b_{n, n}=1 / f(n), b_{n, k}=0$ for $k \geqq n+1$, and $\lim _{n \rightarrow \infty} f(n)=\infty$. For any choice of $f(n)$ so that $\lim _{n \rightarrow \infty} b_{n, n}=0, B=\left(b_{n, k}\right)$ is a strongly regular matrix. The Cesàro transform of a sequence is given by

$$
t_{n}^{\prime}=\frac{s_{1}+\cdots+s_{n}}{n} .
$$

The $B$ transform of a sequence is given by

$$
\tau_{n}=\sum_{k=1}^{n} b_{n, k} s_{k}=\frac{n-1}{n} t_{n-1}^{\prime}+b_{n, n} s_{n} .
$$

It is well known that the Cesàro method sums the Fourier series of any Lebesgue integrable function almost everywhere $[3$, p. 45$]$. We can choose $b_{n, n}$ so that $\lim _{n \rightarrow \infty} b_{n, n}=0$, but $\lim \sup _{n \rightarrow \infty} b_{n, n} s_{n}(x)=\infty$ for the partial sums of Fourier series of functions of the class of our theorem. For such a choice $\left\{\tau_{n}\right\}$ diverges, since $\left\{((n-1) / n) t_{n-1}^{\prime}\right\}$ converges while $\left\{b_{n, n} s_{n}\right\}$ does not converge. In this way we see the class of matrices of our theorem cannot be extended to include all strongly regular matrices. 


\section{REFERENCES}

1. G. G. Lorentz, $A$ contribution to the theory of divergent series, Acta Math. vol. 80 (1948) pp. 167-190.

2. - Direct theorems on methods of summability, Canad. J. Math. vol. 1 (1949) pp. 305-319.

3. A. Zygmund, Trigonometrical series, Warsaw, 1935.

University College of Swansea, Wales 